EPJ Web of Conferences 52, 04010 (2013)

DOI: $10.1051 /$ epjconf/20135204010

(C) Owned by the authors, published by EDP Sciences, 2013

\title{
Looking for Strange Quark Matter in Cosmic Rays
}

\author{
S.B.Shaulov ${ }^{\text {a }}$ S.P.Bezshapov \\ ${ }^{1}$ P.N.Lebedev Physical Institute, Leninsky prospect 53, Moscow 119991, Russia
}

\begin{abstract}
Usually it is supposed that the definition of the CR mass composition in knee region is the key to problem of CR spectrum modification in this range. However tens of the experiments were done for the last half of century and have not decided this problem up to now. The possible causes of fiasco and arguments in favour of necessity to reformulate attack method are discussed, taking into account a new experimental data about fine structure of CR spectrum and EAS core investigations. The possible presence of the exotic processes in the area of a knee is discussed. If exotic component really exists in CR then impossible to formulate correctly more common problem of mass composition without solving this one. It is represented, that the problem of presence of an exotic component in CR should be solved easier than a CR composition problem. The observational basis is discussed. The hypothesis of strange quark matter in CR is suggested for the exotic component.
\end{abstract}

\section{Introduction}

Studying of CR spectrum at superhigh and ultrahigh energies (UHECR) is one of the most important problem of astrophysics. Because of effect of a relict cutoff of $\mathrm{CR}$ spectrum predicted by Grejzen-Zatsepin-Kuzmin (GZKeffect), UHECR sources should be in limits of 30-50 Mpc. In this case at our Galactic or Metagalaxy there should be unknown astronomical objects with a giant energy release. At energies $E>10^{19} \mathrm{eV}$ the gyro radius of protons in the Galactic magnetic field ten times exceeds a thickness of a disk of Galactic, therefore sources should be visible on anisotropy created by them in directions of arrival UHECR. Effects of the last measuring, on the one hand, indicate lack of an cutoff in spectrum UHECR, and with other, on lack of the considerable anisotropy in directions of their arrival. Observable inconsistency can be linked to new physics that requires detailed observational studying of a problem. For this reason the area of GZK-effect $>10^{19} \mathrm{eV}$ represents the special interest. At the same time, the behavior of a spectrum in the area of maximal energies can be bound to the $\mathrm{CR}$ composition originating in the area of a so-called knee $10^{15}-10^{18} \mathrm{eV}$.

Main area of our interests is investigating of fine structure of CR spectrum and EAS cores in the area of a knee, $10^{15}-10^{18} \mathrm{eV}$ by means of different methods used in experiments HADRON and SPHERE.

\section{HADRON and SPHERE arrays.}

The HADRON is a high mountain EAS array which consists of 114 surface scintillation detectors square $0.25 \mathrm{~m}^{2}$ and $1 \mathrm{~m}^{2}$, hodoscope of muon counters under 20 m.w.e.
$\left(E_{\mu}>5 \mathrm{GeV}\right)$ disposed partially directly under a center of installation and in a tunnel in length of 50 meters, hronotron and emulsion chamber (EC) square $162 \mathrm{~m}^{2}$ with four layers of ionization chambers criss-cross. Scintillation detectors placed in circles with radius 16, 20, 40, 55 and $70 \mathrm{~m}$. At center over EC and burst detector 11 stations with three scintillation detectors in everyone are placed. Layout of scintillations and muons counters is shown on figure 1.

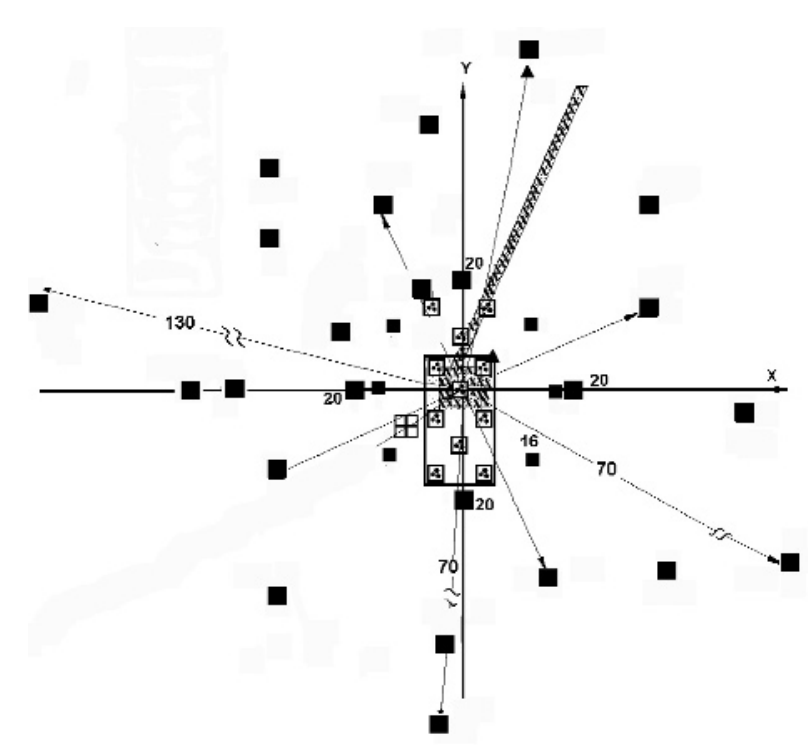

Figure 1. Surface (electrons) and underground (muons) array of HADRON installation. The shaded area shows location of muon detectors in an underground laboratory.

Scheme of EC and burst detector is shown in a figure 2.

\footnotetext{
a. e-mail: ser101@inbox.ru
} 


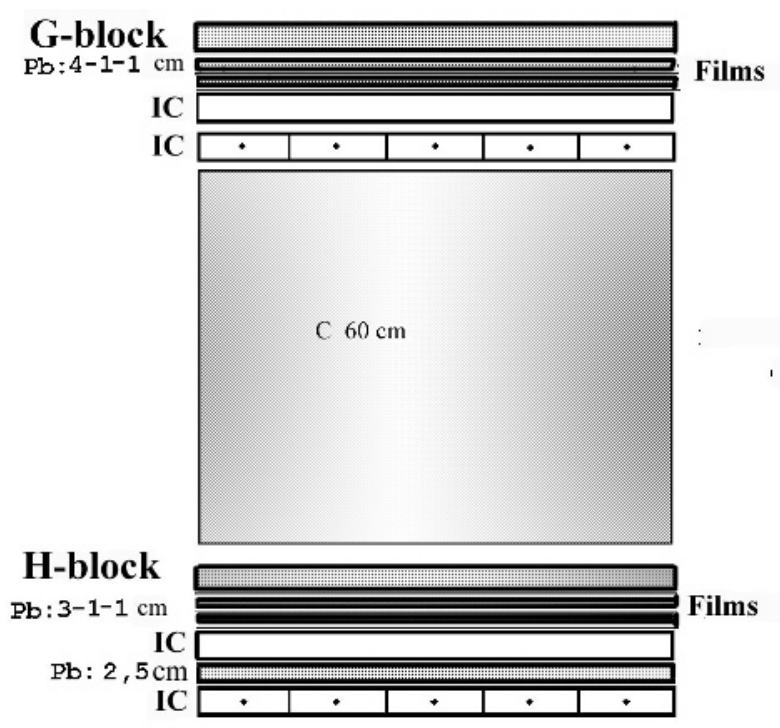

Figure 2. Construction of emulsion chamber and burst detector. The width of ionization chambers $25 \mathrm{~cm}$.

In difference from usual EAS procedure the location of an shower axis was determined by burst detector with much better accuracy of 0.1-0.2 m and angles $\vartheta$ and $\varphi$ were determined by hronotron. The reconstruction of the EAS size $N_{e}$ and "age" parameter $\mathbf{s}$ was performed by means of approximation the experimental densities of charged particles with Nishimura-Kamata-Greisen (NKG) functions. The EAS energies were determined by means formula [1]:

$$
E_{0}=1.51 \cdot 10^{10} N_{e}^{0.84} \mathrm{eV}
$$

High accuracy of EAS axis definition and close-packed arrangement of detectors in the center of the HADRON allows trusty determine LDF of electrons up to distances $\sim 1$ meter from a shower axis. It is especially important for EAS with age parameter $\mathbf{s} \sim 0$. In this case $\mathrm{LDF} \sim 1 / r^{2}$, the integral disperses and $N_{e} \rightarrow \infty$. To eliminate possible distortions of a CR spectrum because of the registration of showers with small values $\mathbf{s}$ flattening procedure was applied to LDF of electrons, i.e. limitation of LDF growth on distances $r<0.5 \mathrm{~m}$. Besides, in method of least squares the quadratic form was used as fallow

$$
F=\sum_{i=1}^{k} \frac{\left(\rho_{i}^{\exp }-\rho_{i}^{\text {theor }}\right)^{2}}{\sigma_{i} \rho_{i}^{\text {theor }}}
$$

where $\rho_{i}^{\exp }$ and $\rho_{i}^{\text {theor }}$-values of the observational and theoretical densities of charge particles in $\mathbf{i}$ detector, and $\sigma_{i}$ - an error of density. The value of $\sigma_{i}$ in a denominator decrease a particular role of terms (detectors) with major densities, i.e. detectors on small distances from EAS axis. Leveling the contribution of terms on small and major distances increments reliability of parameter $\mathbf{s}$ definition, especially in case of small $\mathbf{s}$, and yields the best approximating of experimental data. This alternative can be observed as method of least squares with scales $1 / \sigma_{i}$ in a case when density distributions are determined by a Poisson low: $\mathrm{D}\left(\rho_{i}\right)=\sigma_{i}^{2}=\rho_{i}^{\text {theor }}$ [2].

The installation HADRON was operated in this configuration during 1985-91 years. The installation run with efficiency about $\sim 90 \%$ of a calendar time because of the necessities of comparison film events in EC ( $\gamma$-families) with EAS. The partition of EC and replacement of x-ray films was yielded annually and occupied about one month. On figure 3 the received CR spectrum in comparison with data AGASA is presented [3].

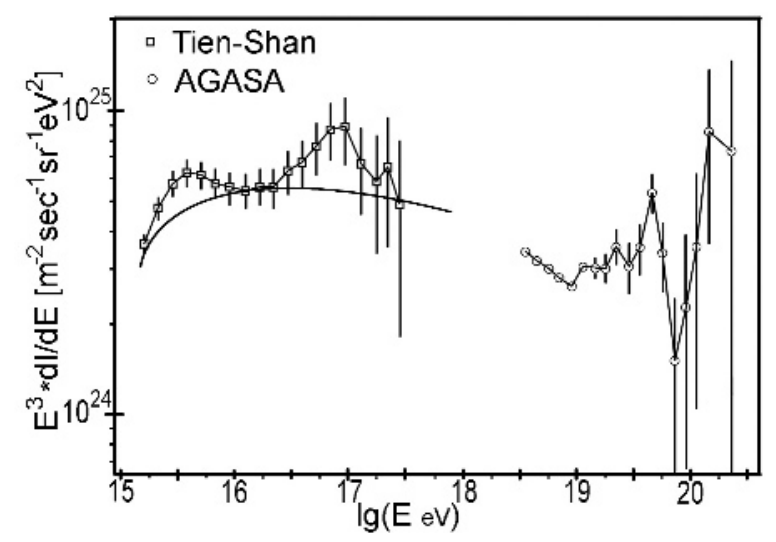

Figure 3. Tien-Shan and AGASA CR spectra.Tien-Shan spectrum (left) divided on two parts.

The spectrum has the unusual form because of bump at energy $100 \mathrm{PeV}$. The break or knee of CR spectrum at energy $3 \mathrm{PeV}$ is traditional and is confirmed by many experiments. The appearance of bump at $100 \mathrm{PeV}$ was unexpected enough and demanded confirmation.

To examine this result by another method we have begun Cherenkov quasi calorimetric experiment SPHERE on Baikal [12]. The measurements were began in 2008. The idea of experiment was suggested by A.E.Chudakov to detect EAS by Cherenkov radiation reflected from a snow ground surface [4]. At rather small mosaic photomultipliers $(\sim 100 \mathrm{PM})$ the diffusion luminous reflectance from snow allows to ensure square of assembly of events proportional to a square of a lifting height of installation $\left(S \sim H^{2}\right)$. Experiment is fulfilled jointly SINP MSU, FIAN and INR of Russian Academy of Sciences over a snow-covered surface of lake Baikal by means of the apparatus lifted on the captive balloon to altitudes $H \approx 1 \mathrm{~km}$. Now there is statistics selection. On figure 4 the working moment of preparation of a night session of measuring is shown.

Preliminary data of SPHERE experiment in comparison with HADRON CR spectrum in the range of 1-1000 $\mathrm{PeV}$ are presented in a figure 5. The energy of HADRON spectrum multiplied by 0.85 to normalized data.

Data of these experiments basically confirm presence of peculiar properties in a spectrum at energies 3 and 100 PeV. Decreasing of energy by $15 \%$ lays within accuracy of energy definition. 


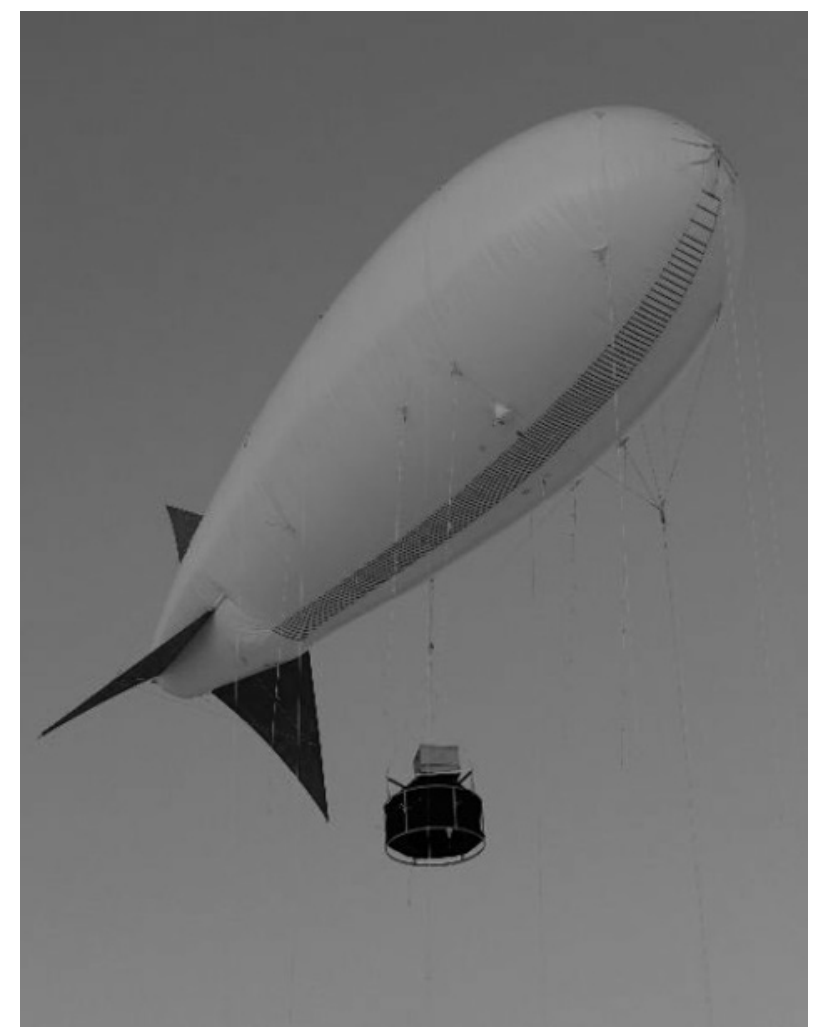

Figure 4. The working moment of preparation of a SPHERE apparatus to a night session of measuring.

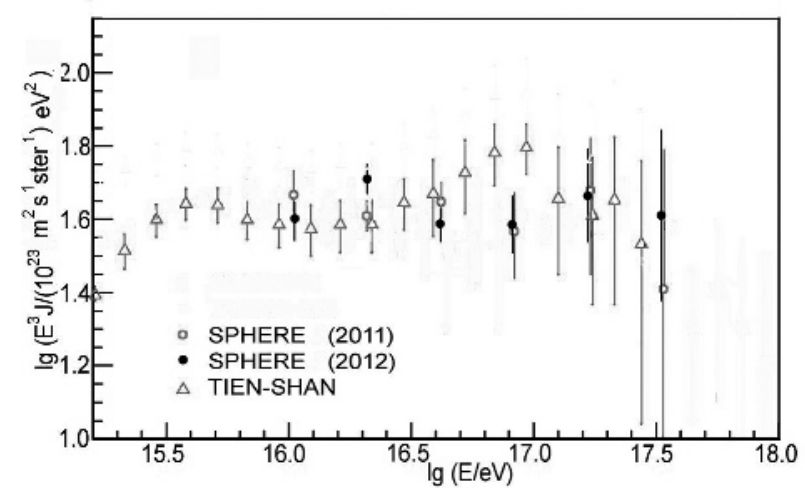

Figure 5. Preliminary CR spectra of SPHERE in comparison with normalized (0.85E) HADRON spectrum at energy range 1$10^{3} \mathrm{PeV}$.

\section{Experiment and the theory}

By present time $\mathrm{CR}$ spectrum is measured in all range $10^{10}-10^{20} \mathrm{eV}$. As a first approximation it has power form practically in all range. It is possible to consider modern researches as the second stage concluded in studying of thin structure of a spectrum, i.e. small deviations from power behaviour which are seeing only in specific representation when spectrum is multiplied by some degree of energy. The spectrum form with bumps represents very important difference from the simple power law which can give the essential help in the decision of a knee problem.
Studying of thin structure of a spectrum has advanced during last twenty years greatly. In a figure 6 it is presented data of many experiments at energy range $E>1$ $\mathrm{PeV}$ [5-9] . The spectra are multiplied by $E^{3}$, therefore all peculiarities are visible especially clearly. Tien-Shan spectrum is normalized (energies are decreased 10\%) such a way that experimental intensities coincide at area $\sim 3$ $\mathrm{PeV}$.

The main difference from the power form of a spectrum should be bound to bump presence at energies 3, 100 $\mathrm{PeV}$ and $30 \mathrm{EeV}\left(3 \cdot 10^{19} \mathrm{eV}\right)$. Difference in energies compounds some orders, therefore hardly it is possible to explain their parentage one cause.

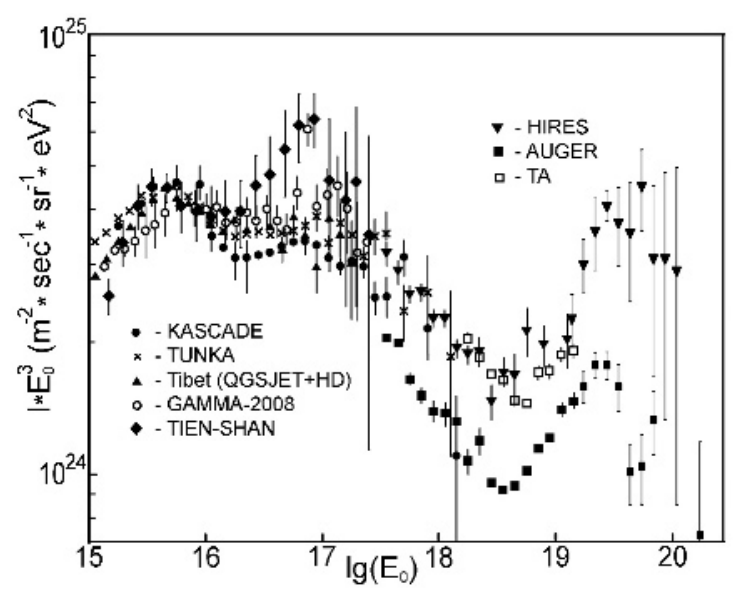

Figure 6. CR spectra measured at different experiments.

Many models of a knee were made for 50 years of investigations but it is possible select only three basic variants. The first one explains magnification of CR spectrum slope at $3 \mathrm{PeV}$ by reaching of the maximum energy of CR acceleration in sources (an accelerating model), the diffusive model bounds it with $\mathrm{CR}$ propagation in Galactic and model of a close single source connects form of CR spectrum at the area of knee with contribution of the close source [10]. Main CR nuclear components must change a spectral indexes at about equal magnetic rigidity $R=E / Z=3 \cdot 10^{15} \mathrm{~V}$ in accelerating and diffusive models. As a result CR weight increase behind a spectrum break at these models.

The modification of CR composition can be connected with behaviour of partial spectrums $N_{e}$ in different intervals parameter $\mathbf{s}$, a method suggested by S.I.Nikolsky. Small s, i.e. more narrow LDF, there should be bound up with light nuclei. Heavy nuclei are disintegrated on nucleons and LDF becomes wider in that case.

Data about CR composition at area $100 \mathrm{PeV}$ bump which were received at Tien-Shan HADRON experiment are shown at figure 7 [17]. Bump at energy $100 \mathrm{PeV}\left(10^{17}\right.$ $\mathrm{eV}$ ) is formed mainly by showers with narrow lateral distribution function $(\mathbf{s}<0.4)$ and rather old showers with $\mathbf{s}>1.2$. The average value of $\mathrm{s}$ thus has quite normal value $\langle\mathbf{s}>=0.6-0.8$. The most striking feature here is that showers with $\mathbf{s}<0.4$ practically miss in simulation calculations. So their emersion in the observational 


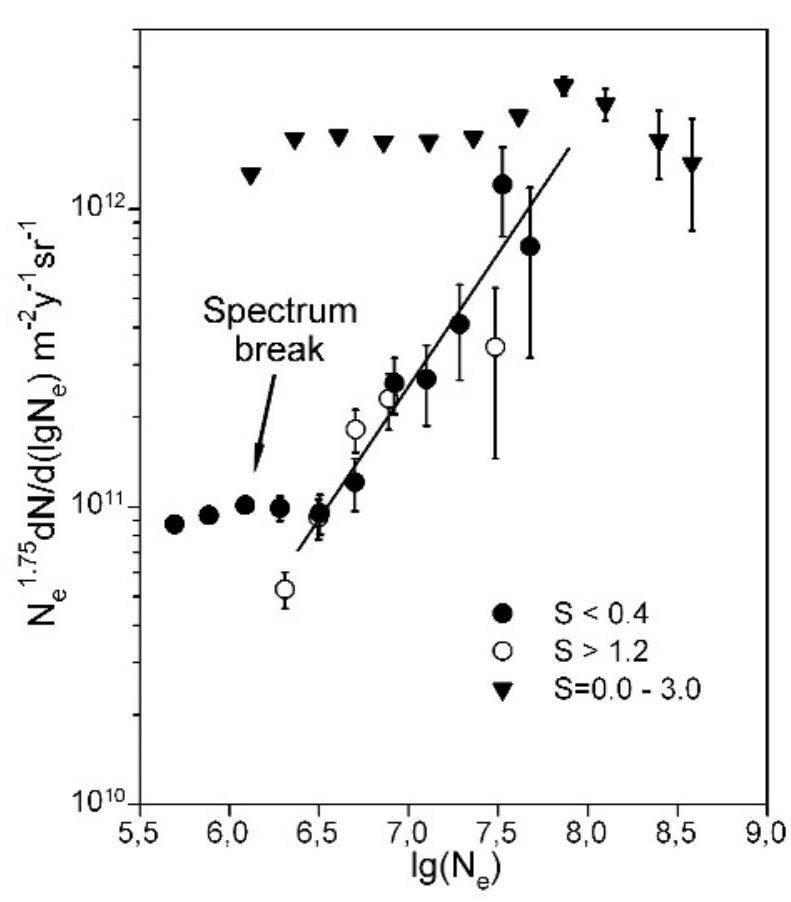

Figure 7. $N_{e}$ spectra for EAS, "young" EAS with $\mathbf{s}<0.4$ and "old" EAS with $\mathbf{s}>1.2$.

statistics necessary to consider as exotic. The part of such showers increases in $\sim 5-10$ times almost at once behind a break of CR spectrum. It contradicts to CR composition weighting in this area, predicted both by accelerating, and diffusion models because growth of showers part with narrow LDF rather indicates increase of light nuclei part ( $p$ and $\mathrm{He}$ ) behind a break.

Other singularity of a spectrum, repeatedly marked in different experiments, is unusual sharpness of a slope modification at energy $3 \mathrm{PeV}$. Neither the accelerating, nor diffusion models cannot explain such shape of a break. It is practically obvious, that if the knee is formed by million sources in Galactic a range of declination of CR spectrum should be greatly spread. On the contrary it is quite natural in model of a single close source. Therefore we will consider further, that the spectrum in the area of a knee is presented by two-component model as it is shown in figure 3 . The basic part of radiation with the smooth modification of a spectrum slope (full curve) is presented by the accelerating, or the diffusion model. But bumps in a spectrum at energies 3 and $100 \mathrm{PeV}$ are formed by single close source.

Difference of data of various experiments in the area around of $100 \mathrm{PeV}$ can arise because of the considerable difference in registration conditions for "young" showers. Tien-Shan spectrum shows the most striking bump may be because these conditions in experiment HADRON for registration of narrow LDF were essentially more congenial. The EAS core was determined with high accuracy 10-20 $\mathrm{cm}$ by means of ionization chambers allocated criss-cross and electronics dynamic range was large enough $2 \cdot 10^{4}$ value. At distances between detectors $7 \mathrm{~m}$ these condi- tions allowed to measure electrons LDF up to distances $\sim 1 \mathrm{~m}$.

In the area of energies $>10^{19} \mathrm{eV}$ recent experiments HiRes and Auger [5] and TA were confirmed the data of experiment AGASA about existence of showers with energy $\sim 10^{20} \mathrm{eV}$. All experiments show bump at $10^{19}-10^{20}$ $\mathrm{eV}$ in figure 6.

It is possible only to guess about further spectrum behavior. Strictly speaking, the problem of GZK cutoff remained, but has transferred in range of energies above $10^{20} \mathrm{eV}$. Of cause many believe that energy after bump $\sim 10^{20} \mathrm{eV}$ is a CR spectrum cutoff but formally the spectrum in this range can be prolonged with the same declination $\gamma \approx-3$ too. Experiment should solve this. From existing designs only probably satellite designs EUSO and LORD have large enough geometric factor $S \Omega$ for obtaining of sufficient statistic at area $>10^{20} \mathrm{eV}$ and can resolving GZK-effect problem experimentally.

The indirect measuring of atomic weight $(\ln \mathrm{A})$ of primary nuclei in figure 8 would seem confirm behaviour suggested by accelerating and diffusive models [11]. But it seams that another interpretation of these data is possible too. The bumps in CR spectrum $\left(10^{17}\right.$ and $\left.3 \cdot 10^{19}\right)$ correlate with relatively large value of $\ln \mathrm{A}$, i.e. with EAS produced by heavy nuclei. It's very strange and not simple situation because contradicts to GZK-effect idea. If photo-nuclei processes of nuclei interaction with relict $\gamma$-radiation play important role then all nuclei have disintegrated for energy range $>10^{19} \mathrm{eV}$. In this case expected that $\ln A \simeq 0$, but instead it $\ln \mathrm{A}$ is increases in the area of bump, i.e. composition is weighted in inconsistency with model of GZK - effect as it is shown on figure 8.

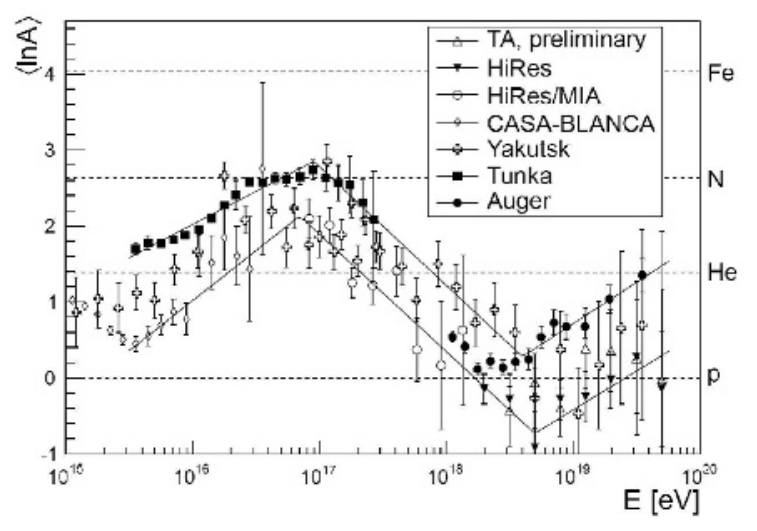

Figure 8. Energy dependence of $\ln$ A for EAS (from [11]).

\section{Fiasco in determination of CR composition.}

From beginning EAS investigations it was understood that main information about CR composition and interaction characteristics is enclosed at hadron spectra of EAS core. Ionization calorimeters permit investigate hadron cores of 
EAS but only them longitudinal development. Study of the hadron spectrums is possible by means of the single detector - X-ray emulsion chamber. Such possibility gives the unique space resolution of EC ( 100 microns) and possibility of energy measuring of individual cascades of $\gamma$ quanta (hadrons). Such a way energy spectra of $\gamma$-quanta in EAS with different energies were received. Furthermore two Ne spectra for all EAS and EAS with $\gamma$-quanta $(\gamma$ families) were received. These two spectra should be described by one model, that sharply limits possible variants. The elementary model which allows to approximate both spectra simultaneously was used [22]. In the inference we will show the possible cause of why the problem of CR composition in the region of knee is not solved till now.

Presence of bumps at energies 3 and $100 \mathrm{PeV}$ in a spectrum of all particles guesses their presence in individual spectrums of various groups of nuclei. It proves to be true by experiment HADRON data about EAS spectrum with $\gamma$-families shown at figure 9 .

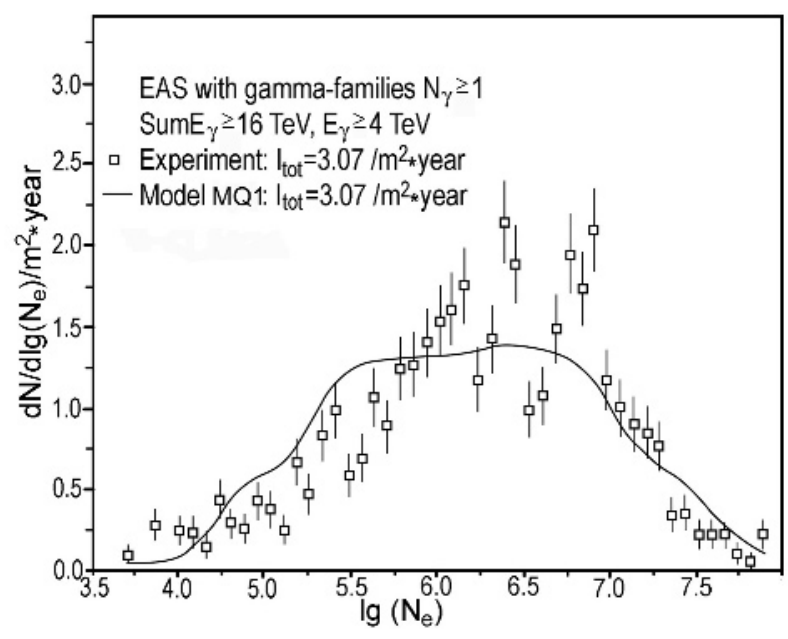

Figure 9. Experimental and theoretician spectra of EAS with $\gamma$-families.

The spectrum received in experiment essentially differs from theoretical one by presence of several peaks which should be referred to various groups of nuclei [13].

Within the limits of the elementary model without fluctuations in which spectra of all groups of nuclei are similar and have on two bumps at magnetic rigidity $\mathrm{R}=\mathrm{E} / \mathrm{Z}=3$ and $100 \mathrm{PV}$, it is offered to look to what consequences results bumps presence in a spectrum of all particles. Individual nuclei spectra were normalized on experimental data of direct measurements as it is shown at figure 10. Presented intensity are counting on one nucleon of a nuclei (n).

Results of modeling approximation of two experimental $N_{e}$-spectra: for all EAS and for EAS with $\gamma$-families are shown at figure 11. The consent of the observational and theoretical spectra quite satisfactory

Individual energy spectra of various groups of nuclei and approximating of the total CR energy spectrum are presented on a figure 12 .

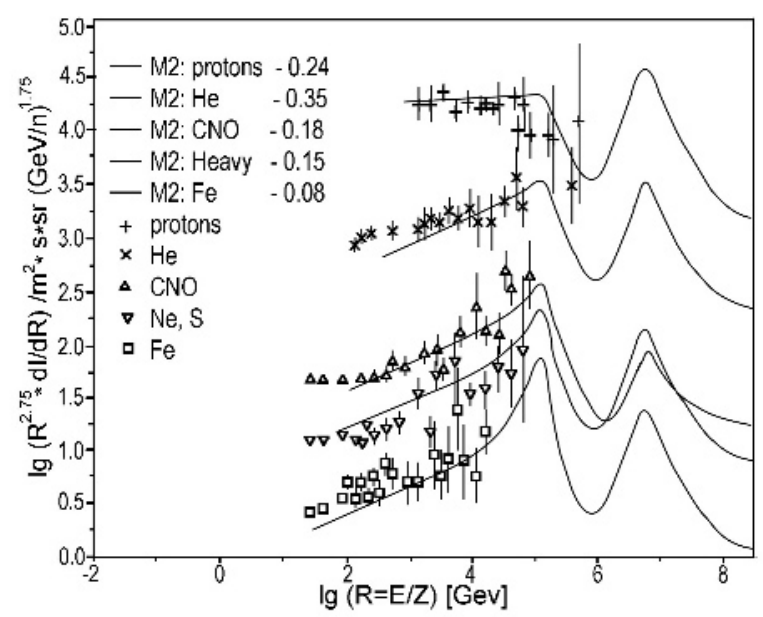

Figure 10. Modeling spectra of different nuclei (solid lines) normalized at data of direct measurements (points). Intensity are counting on one nucleon of a nuclei (n).

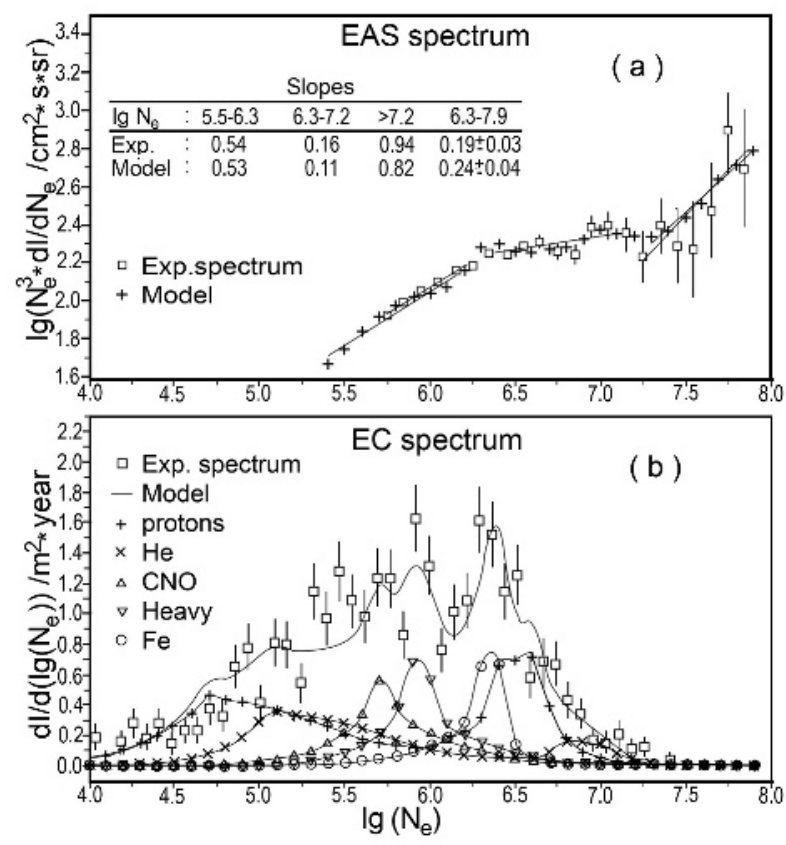

Figure 11. Modeling approximation of Ne-spectra: a - spectrum of all EAS; $b$ - spectrum EAS with $\gamma$-families; points-experiment, solid lines - model.

As follows from a figure 12 because of bumps in $\mathrm{CR}$ spectrum the oscillations of a CR composition in energy spectrum exceeds energy resolution of EAS method $(\sim 30 \%)$. The capability of measuring of CR composition by EAS method in the interval energies $10^{15}-10^{18} \mathrm{eV}$ is doubtful because of this cause. May be it is a main reason of fiasco in CR composition determination during last fifty years.

The presented form of spectrums does not mean, that the composition basically cannot be measured, but means, that it will be different for different installations. Of cause to measure right $\mathrm{CR}$ composition is possible, but in a cos- 


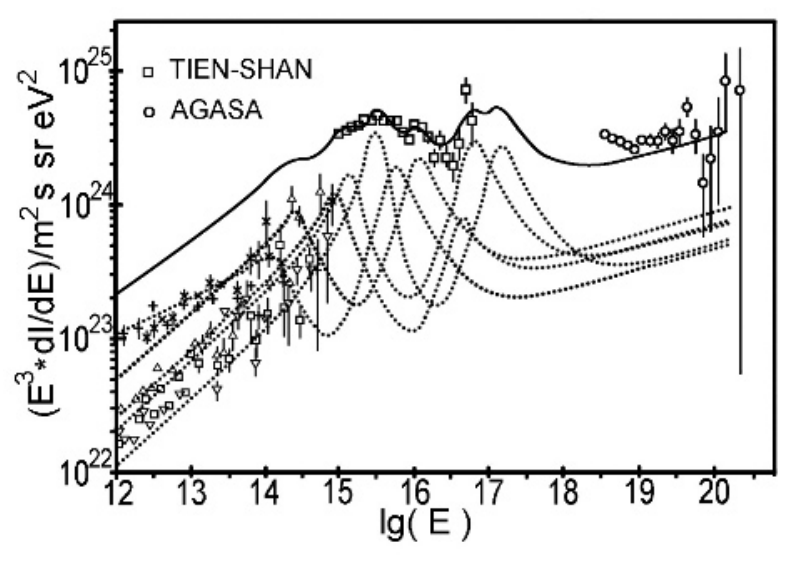

Figure 12. Individual energy spectra of nuclei groups and approximating of the total $\mathrm{CR}$ spectrum.

mos by direct methods, for example, by means of the installation allocated on the moon.

If it is right, then it is a bad news because always was considered, that exactly CR composition is that key which will allow to discover the cause of a parentage of a knee. If it cannot be measured, what remains? More interesting and possible even more simple alternative is bound to availability of existence in a primary cosmic rays the new component which is distinct from nuclei, for example, corpuscles of strange quark matter.

\section{Exotic in cosmic rays.}

Though formally two-component model presents a good approximation of the real spectrum form in knee area there are a number of unresolved problems which call for a more close look. Some experimental results, basically received on high mountain stations force to introduce exotic explanations of them, in particular presence of the new CR component such as corpuscles of strange quark matter (SQM) at range of the knee. Certainly from experiment immediately does not follow, that exotic CR component, if exists, it is corpuscles of SQM. We use this hypothesis as some comprehensible alternative, which can have an astrophysical substantiation (quark stars), explicitly does not contradict EAS data and admits check.

A number of results received in experiments on TienShan which should be referred to exotic effects are presented below.

(a) First of all a hadron long flying component of EAS was detected by means of a big ionization calorimeter [15]. The hadron absorption length is increased twice from 500 to $1000 \mathrm{~g} / \mathrm{cm}^{2}$ in the area of a CR spectrum break (at $E=$ $3 \cdot 10^{15} \mathrm{eV}$ );

(b) The absorption curve of high energy $\gamma$-quanta $\left(\pi^{0}\right)$ in an atmosphere is shown in a figure 13, which was received by compilation of various emulsion chamber experiments. This curve may be presented as the sum of two curves where one of them has usual absorption length $\lambda_{\text {att }} \simeq 90 \mathrm{~g} / \mathrm{cm}^{2}$ and another (abnormal) has $\lambda_{\mathrm{att}} \simeq 250 \mathrm{~g} / \mathrm{cm}^{2}[16] ;$

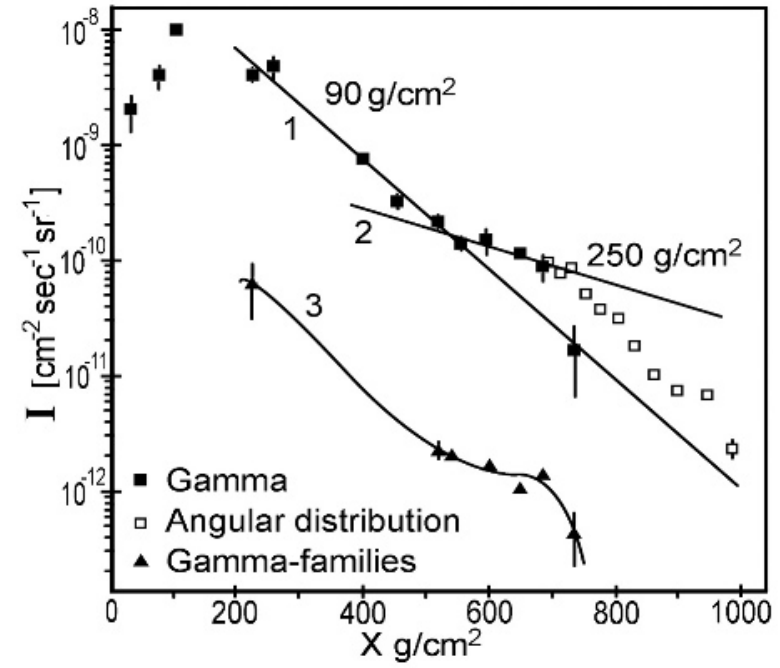

Figure 13. The absorption curve in atmosphere for $\gamma$-quanta and $\gamma$-families.

(c) Anomalously narrow EAS with age parameters $\mathbf{s}<0.4$ are practically absent in the model calculations, i.e. their presence at experimental events in itself is exotic. Besides just such showers with abnormal narrow lateral distribution functions (LDF) of electrons form the second bump in a figure 3 at $E=10^{17} \mathrm{eV}$;

(d) Two bumps in Ne spectrum may be explained as a contribution of two different close sources, however the hypothesis of SQM allows to manage with only one source (quark star). In this case two bumps in Ne spectrum can be derived from one bump at energy spectrum because of big difference of total number of charged particles in cases when primary strangelet has disintegrated on hundreds hyperons over installation and has attained it without disintegration;

(e) EC usage as EAS core detector in hybrid experiment HADRON [17] has allowed receiving $N_{e}$ dependencies for muon and hadron components of EAS with $\gamma$-families. These dependencies contradict each other if primary particles are nuclei. In a figure 14a) the $N_{e}$ dependencies of an average muon number for all EAS and EAS with $\gamma$-families are shown [18]. As EAS with $\gamma$-families considered to be proton EAS mainly, their muon number has to be smaller than for all EAS (dotted line), but experiment shows an opposite result. This seems contrary to any EAS models if primary particles are nuclei. This contradiction does not depend on an interacting and composition CR model.

Moreover muon data come into open conflict with $\gamma$ quanta energy spectra. These spectra may be considered as hadron spectra because main channel of $\gamma$-quanta generation is $\pi^{0} \rightarrow 2 \gamma$. As shown by figure 14b) energy spectrum of $\gamma$-quanta become more hard for $N_{e}>10^{6}$. It follows from model calculations, that if the index of spectrum slope $\mathrm{b}\left(E_{\gamma}^{-b}\right)$ takes the value $\mathrm{b}=2$ it means pure iron in composition of primary $\mathrm{CR}$ and $\mathrm{b}=1$ for pure protons. Really value of $b$ decreases from $b=1.8$ (mix composition) 


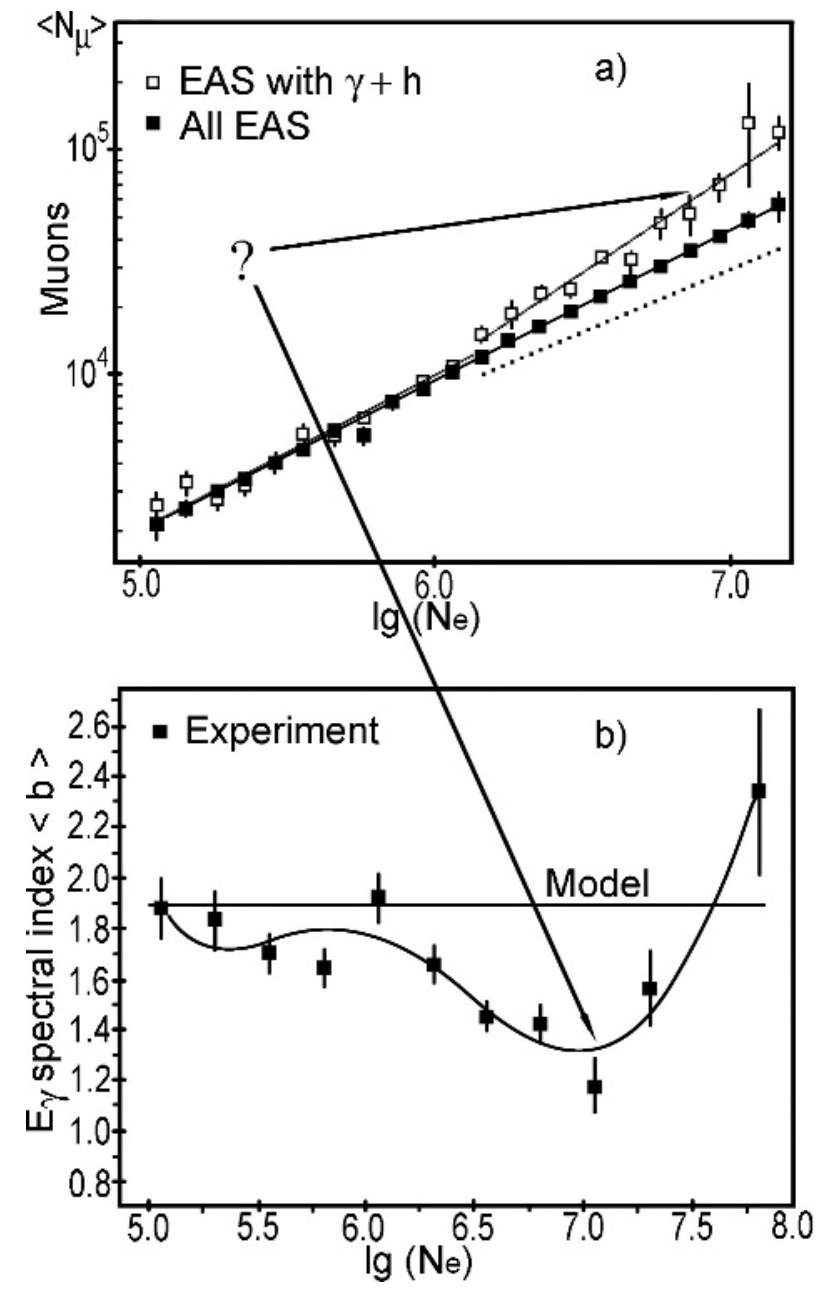

Figure 14. a) $N_{e}$ dependence of an average muon number for all EAS and EAS with $\gamma$-families; b) $N_{e}$ dependence of slope b $\left(E_{\gamma}^{-b}\right)$ at energy spectrum of $\gamma$-quanta.

down to $b=1.2$ (almost purely proton) and in that case energy dissipation in the atmosphere above detecting level has to decrease with $N_{e}$.

The changes in CR composition or in EAS model are recuired in order that to resolve this contradiction. The first variant is represented more preferable, therefore we enter hypothesis SQM in cosmic rays.

\section{Discussion}

The presence capability of an abnormal CR component cardinally changes the approach to planning of groundbased experiments. As a rule solution of a knee problem one's see in definition of CR nuclear composition and its dependence from a primary energy. The determination of muon component yields main method of CR composition definition. However, as follows from the previous data, the behaviour of muon component in the knee region essentially differs from the model one. It is possible to tell the same about behavior of $\gamma$-quanta with energies a few $\mathrm{TeV}$ and more.More hard hadron spectra behind a break $\left(N_{e} \simeq 1.5 \cdot 10^{6}\right)$ contradicts $\mathrm{CR}$ weight increase in this range. Escaping of this situation can be bound to the additional component in two-component CR model, assuming that it is responsible for all anomalous effects. Such version basically is possible with assumption, for example, that the close source is quark star and flux of SQM particles goes from it. The idea of SQM in CR has been suggested earlier in [19] for an explanation of CR events of type of the centaurs which have been detected at Mt. Chacaltaya in emulsion experiment of Brazil-Japan collaboration [20].

The locality of such component limiting its presence only by knee region can be explained by meta stability of SQM corpuscles. If the time of their life is restricted by an order value $\tau \simeq 10^{6}$ years as suggested in [21] the investment in CR flow will be yielded only by the most proximate sources, and is possible only one source. The offered hypothesis is based on experimental results, but is exotic enough and should be examined. EAS check of this kind is possible only on the basis of detailed studying of EAS core.

The unique detector, allowing adequately solving this problem is EC exposed as EAS core detector. New high mountain EAS installation with EC and muon detector big enough square $\left(\sim 10^{3} \mathrm{~m}^{2}\right)$ would allow to advance in range of energies up to hundreds $\mathrm{PeV}$. The interval above $10 \mathrm{PeV}$ seams a most informative because effectiveness of $\gamma$-families generation in EAS becomes nearer to $100 \%$ for these energies. Because of this EC exposition must exceed thousands $\mathrm{m}^{2}$.

\section{The conclusion.}

The search of the exotic CR component (or the demonstration of its absence) has to be the main goal of the new experiments. The minimal composition of future complex EAS installation has be as fallow: EAS-detector (electrons), $\mu$-detector (muons) and emulsion chamber as EAS core-detector can resolve this problem. Undoubtedly, installation addition with other subsystems only will strengthen it's potential.

\section{Acknowledgements}

We are thankful to all participants of experiment HADRON and particular Dr. Cherdyntceva K.V and Janseitova J.K., who processed all emulsion data. The participation in symposium became possible thanks to financial support of the Program of Presidium of the Russian Academy of Sciences "Fundamental states of materia and the astrophysics".

\section{References}

[1] A.D.Erlykin, diss. Multivariate analysis of hadron cascades in the atmosphere for nuclearl and astrophysical investigations of cosmic rays, Dr.Ph.-M.Nauk, FIAN, Moscow (1986) (in russian).

[2] S.B.Shaulov, diss. The investigation of CR composition in area $0.1-10 \mathrm{PeV}$ by gibrid method of EAS and $\gamma$-families detecting., FIAN, Moscow (1999) (in Russian). 
[3] Shaulov S.B., Proc. 27 Int.Cosmic Ray Conf., Hamburg (2001) 277.

[4] A.E.Chudakov, Experimental methods of studying cosmic reays of ultrahigh energies, Proceedings of the All-Union Symposium, Yakutsk (1972) 69 .

[5] Abraham J et al. Phys. Rev. Lett. 101 (2008) 061.

[6] S.B.Shaulov and S.P.Bezshapov, ECRS, Moscow (2012) Jornal of Physics, to be published.

[7] T.Dzhatdoev, SPHERE collaboration, ECRS, Moscow (2012) Jornal of Physics, to be published.

[8] L.A. Kuzmichev, TUNKA collaboration, ECRS, Moscow (2012) Jornal of Physics, to be published.

[9] A. Garyaka. , GAMMA collaboration, ECRS, Moscow,2012, Jornal of Physics, to be published.

[10] Erlykin A D and Wolfendale A W, J. Phys. G 23 (1997) 979-989.

[11] K.-H.Kampert, M.Unger, APh, 35 (2012) 660.

[12] Antonov R.A., Anyuhina A.M., Bonvech E.A. et al., Nuclear Physics B - Proceedings Supplements, 175176 (2008) 194-197.
[13] S.B.Shaulov, Preprint FIAN N8, Moscow (1998) 330 (in Russian).

[14] A.D.Erlykin, A.W.Wolfendale, Nucl.Phys.B, Proc.Suppl., 175-176 (2008) 186-189.

[15] Yakovlev V I, AIP Conf. Proc. 146 (1993) 432.

[16] Janseitova J., Nikitin N.V., Cherdyntceva K.V. and Shaulov S.B., Preprint FIAN N10 (1999) 1-30 (in Russian).

[17] Shaulov S B, Problems of atomic science and technology 3(29) (1986) 72 (in Russian).

[18] S.B. Shaulov, Heavy Ion Physics 4 (1996) 403-422.

[19] J.D.Bjorken and L.D.McLerran, Phys. Rev. D, 20 N9, (1979) 2353-2360.

[20] C.M.G. Lattes, Y. Fujimoto and S. Hasegawa,Phys. Rep. 65 (1980) 151.

[21] E. Keith, E. Ma, Heavy Ion Physics 4 (1996) 381386.

[22] Shaulov S.B., Preprint FIAN N8 (1999) 3 (in Russian). 